\title{
Elements of Categorical Logic: Fifty Years Later
}

\author{
Valeria de Paiva and Andrei Rodin
}

This volume of the Journal Logica Universalis collects together papers on categorical logic in an extended sense. The idea for this volume arose out of the workshop on categorical logic associated with the conference Unilog 2010 held in Lisbon in June 2010. We feel that many of the ongoing activities in this exciting and somehow under-represented research area should be presented to a wider audience in the form of a special journal issue. In view of the diversity of the topic a general call for papers was issued. Papers presented at the workshop as well as new papers have been submitted and reviewed, following the usual criteria of this journal.

The Topic. Since our topic is a interdisciplinary subfield of logic, computer science and philosophy we believe that we should as least discuss some questions that the researchers interested in categorical logic might pose themselves.

- What do we mean by categorical logic?

- What are the boundaries of this subfield? Is it an emergent one?

- Which are the important problems in this area?

- Why should any one pursue a programme of investigation in this area?

- Can we see ways of increasing the interaction between the largely parallel communities (mathematicians, traditional logicians and computer scientists) involved?

- Most importantly, what are the trends for the future?

The aim of this preface is not to answer these questions, but simply to give any interested researcher a few pointers to the literature since the questions are hard, several answers are possible and many are already addressed in the literature.

To begin with, we need to discuss at least a little what we mean by "categorical logic". There are many pre-theoretical definitions available and several non-equivalent definitions are reasonable. Marquis and Reyes [18] say, for example, that "categorical logic is algebraic logic, pure and simple". But algebraic logic itself requires explanation and there is a need for circumscribing the notion, as there are many different research programs that could be considered algebraic logic. Since categorical logic also involves a fruitful interplay 
with algebraic geometry, in particular, through Topos theory and Homotopy Type theory, it can be equally described as "geometrical" in a broad sense of the word (Geometric logic in the established narrow sense of the term [23] also falls naturally under the scope of categorical logic). Thus very different research frameworks can be encompassed under the umbrella of categorical logic.

Both syntax and semantics of logical systems can be given categorical treatments. Going further, logic is traditionally divided into proof theory, model theory, recursion theory and set theory, and considering category theory as a methodology, that can be applied to any of these four areas. And indeed it has been applied, with different degrees of success to the four traditional branches of logic and even to the less traditional, but very upcoming subject of logical complexity.

However using category theory as a tool or a methodology for treating old and new logical problems does not in fact exhausts today's categorical logic. For categorical logic also results from important internal developments in category theory, algebraic geometry, functional analysis, algebraic topology and related fields [14]. We mean here Lawvere's advancement of the logical significance of category-theoretic constructions as adjoint functors (quantifiers as adjoints to the substitution functor), of Grothendieck topologies (topology as a modal operator), of Cartesian closedness of certain functional spaces and similar examples. Such approach to logic "from within" a category (which by its intended interpretation may be not "logical" but, for example, geometrical as in the case of toposes) can be opposed to the more traditional notion of logic as an external framework for building mathematical theories.

Most of the work to be discussed in this preface will be concerned with categorical proof theory and categorical model theory. Both started in the late sixties with the work of Lawvere, Joyal and Lambek. There are learned discussions on the history of categorical logic in [1] and [18]. For the purposes of our discussion here, we take the year of 1963 (Lawvere completed his Ph.D. [11] at Columbia University in 1963 with Eilenberg) as the date to starting counting "categorical logic" from. As far as categorical model theory is concerned it suffices to say that doing mathematical model theory using functors as models, instead of sets, the purvey of categorical model theory does bring about many of the rewards of abstraction. But it is the approach that considers morphisms of a category as proofs in logic, the so-called categorical proof theory that mostly interest us here. Categorical proof theory has also been called "categorical type theory" in at least one influential book [10].

The realization that there is a structural unity of three things: (i) proofs in Natural Deduction, (ii) certain kinds of terms in lambda-calculus and (iii) morphisms in appropriate kinds of categories (sometimes called the extended Curry-Howard isomorphism), is one of the most striking results in mathematics, logic and computer science from the last century. This Curry-Howard correspondence was slowly uncovered through the years via the work of many people (starting with Schönfinkel, Curry, Howard, Prawitz, Tait, Lambek and Lawvere) and despite the many articles and books $[3,22]$ written about it, its importance has not permeated sufficiently through the communities involved. 
Some of the researchers in categorical logic feel that the relationship between logics and their associated type theories - via extensions of the CurryHoward isomorphism - is the main goal of categorical logic, while others are more interested in the affordances of the categorical methods as far as algebraic logic is concerned, either for specific areas of application or for general philosophical and mathematical considerations. A somewhat whimsical description of the former can be found in R. Harper's The Holy Trinity. ${ }^{1}$

Amongst the philosophical considerations sits squarely the controversy on whether categories are an adequate alternative foundation for Mathematics, as proposed by Lawvere or not. The subject is fascinating and challenging, but we will eschew it in this short preface. One of us editors has much more to say on the subject [21] and the other has no views on this polarized issue. Two of the papers in this volume (by Maietti and Rosolini and by Johnstone) can be seen as contributions to this discussion.

Similarly there is great controversy on the need for and best formulation of higher-order category theory in general and in connection with categorical logic, in particular, whether related to applications in theoretical physics or not. The online forum The n-Category Café and associated wiki, the $\mathrm{nLab}^{2}$ have been showcasing the work on this growing branch. One of the papers in this volume (by Jacobs) hints at this controversy, but eschews it in an attempt to be understandable to more researchers.

A smaller controversy (or perhaps simply a lack of communication) surrounds the model theoretic work on Institutions (conceived by its originators as a categorical model theory for computer scientists [9]) from the work on categorical proof theory for computer scientists, in the spirit of Martin-Lóf's type theory and the wealth of work originating from that. Duval's contribution to this volume sits on the side of model-theoretic work, making bridges to other more concurrency inspired work on graphs.

Contexts and Prospects. Categorical logic may appear as a very special subdivision of modern mathematical logic, of which there are so many nowadays. However categorical logic has a least one feature, which makes it generally more substantive than other logical characterizations. This concerns the idea of internal logic (of an appropriate category), which has been already mentioned above. There is reasonable hope that this approach using internal logics may bridge the existing gap between mathematical logic and the rest of mathematics. Despite the fact that modern logic widely uses mathematical methods (in particular, for proving meta-theorems), mainstream mathematics very rarely applies any logical methods or logical considerations explicitly. Thus mathematical logic and its related disciplines (such as axiomatic Set theory) look like relatively isolated pieces of today's mathematics. This is, quite obviously, at odds with the traditional understanding of logic as a universal tool for strict

\footnotetext{
${ }^{1}$ http://existentialtype.wordpress.com/2011/03/27/the-holy-trinity/.

${ }^{2}$ http://ncatlab.org/nlab/show/HomePage.
} 
reasoning and particularly for mathematical reasoning. When methods of categorical logic help one make explicit logical structures associated to categories, which are important on their own (like homotopical categories [5]), studying these logical structures is no longer separate from studying other aspects of the given categories. In these cases logical methods serve the purpose of internal organization of mathematical reasoning rather than its external regimentation.

Thus it would be certainly wrong to think of categorical logic as a specialized logic like, say, tense logic, which treats this or that specific form of reasoning. The scope of categorical logic is not limited in that respect. It is more adequate to describe categorical logic as logic, which uses a set of specific mathematical techniques, namely the techniques of Category theory. However one should bear in mind that the choice of these techniques is not merely pragmatic because it prompts a significant rethinking of the role of logic in mathematics and elsewhere and makes (categorical) logic into a proper part of today's mathematics and computer science.

Finally we should say that one can consider categorical logic as a tentative version of an Universal Logic. When doing that we have in mind not some all-embracing system of logic but rather a toolkit, which allows for building integrated logical systems from their elements. This way the category-theoretic setting helps to connect logic to the rest of mathematics and science, and thus makes logical methods more useful in these fields. Although none of the papers published in this volume treats directly the issues of unification in logic and application of logic in mathematics and science, we believe that these contributions serve these longer-term goals as well.

The Papers. The contributions in this special volume of the Journal Logica Universalis exemplify developments of some of the lines of research mentioned above.

We start the volume with classical categorical logic. More than 30 years ago Peter Freyd constructed certain Grothendieck toposes which were supposed to demonstrate the independence of the axiom of choice from set theory [8]. The exact sense in which that claim is true has remained somewhat obscure to the general public. Peter Johnstone's paper in this collection provides a new clarification of these results as indicated by its title "what do Freyd's toposes classify"? Freyd's original proof relies on Fourman's observation that there is an interpretation of the first-order language of set-theory, making all the axioms of ZF true in an arbitrary Boolean Grothendieck topos. Thus to prove the independence of the axiom of choice from set theory, it is enough to construct a topos containing a counterexample to the topos-theoretic axiom of choice in its part described by the Fourman interpretation. Freyd gave a generic method for constructing such toposes and gave two particular examples that Johnstone's paper investigates, from the perspective of considering them as classifying toposes, that is from the perspective of the geometric theories whose generic models they contain. Johnstone's change in perspective, going for the geometric theories behind the counterexample toposes, instead of looking at them from the point of view of set-theoretic forcing (as in the extensive study by Blass 
and Scedrov [2]) seems a good way to rekindle interest in the problems of the correspondence between topos-theoretic set theory and naive set theory. The investigation of this correspondence, started by MacLane and Moerdijk [19] would result in a better understanding of what is set theory. Lawvere's 1964 observation [12] that a morphism between toposes that satisfy too many setlike restrictions must be an equivalence, indicates the delicacy of the question.

There are various foundations for constructive mathematics available in the literature and their inter-relations are not as clear as one might wish. The paper by Maietti and Rosolini looks at the problem of providing categorical constructions to formalize the foundations for constructive mathematics. Their perspective is that ideally, a foundation for constructive mathematics should be at the same time a set theory, in which we formalize mathematical theorems, but also a programming language in which we can extract the computational contents of mathematical proofs. Because one wants this ideal foundation to model extensional features used in the everyday practice of mathematics such as extensional equality of sets or of functions, or quotient constructions, and these notions are incompatible with usual type theory, one solution adopted in practice (and suggested by the first author in previous work) is to provide a two-level foundation where one level is used for program extraction and the other to formalize mathematics. If such a two-level model is chosen, an adequacy condition to the effect that one can interpret the extensional level in the intensional level by means of a quotient completion of the intensional level is required. So one must provide a general categorical notion of quotient completion, a universal construction that "freely adds" quotients for the equivalence relations without adding any further power to the underlying logic. This paper studies such extension by quotients from a different point of view from the traditional "exact completions" in category theory, namely that of the hyperdoctrines (or fibrations) originally proposed by Lawvere for the semantics of type systems [13]. The authors identify the correct notion of a hyperdoctrine with "good" quotients, and show that there is a free completion construction under such quotients. Some results from the theory of exact completions (e.g. weak limits become limits) are seen to hold in this setting, too. The move away from the usual 1-categorical setting to fibrations is sensible, since the semantics for systems of dependent type theory have been seen to be better treated in the fibrational setting.

Jacobs' article introduces a category-theoretic abstraction of the notion of "comparison relation", establishes general properties of this notion and gives a rich list of examples with an eye towards applications in categorical quantum theory, one of the areas of categorical logic growing at fast pace. Large parts of quantum mechanics and quantum computation can be naturally formulated in the theory of dagger-categories and "tame relations" were introduced in the construction of a particular (monoidal) dagger-category of formal distributions. Jacobs introduces a general context in which this notion of tameness makes sense and describes it from a categorical perspective. As mentioned in the paper, the notion of "comparison relation" which is introduced, and the main constructions surrounding it, are a variation of the classical notion of an 
enriched profunctor. It is well-known that enriched profunctors form a generalization of the notion of relations, and the existence of adjoints (daggers) for (enriched) functors regarded as profunctors (which are the "tame" relations in the present article) has a long tradition in categorical quantum physics [6]. But this similarity with profunctors is not pursued in this paper, which aims to be more concrete and to stay at the level of 1-categories.

Duval's contribution starts investigating a correspondence between two formalisms, namely graph rewriting systems and inference in her own "diagrammatic logic". Graph rewriting systems were introduced by Corradini et al. [4]. The left and right sides of a rewrite rule are formalized as graph morphisms and application of the rule is defined using a pushout construction. Diagrammatic logic is a framework devised by Duval based on an abstract view of the consequence-closure operator: a logic is formalized as a functor $L: S \rightarrow T$ where $S$ is seen as a category of specifications and $T$ as a category of theories. The intuitive motivation for this definition comes from the fact that a theory for a given logic is a class of sentences which is saturated: it is a class of theorems from which no new theorem can be derived inside the given logic. For instance, the theory of groups in equational logic is made of all the theorems which can be proved about groups, starting from the usual equational axioms for groups and using only the congruence properties of equality. A class of axioms generating a theory is often called a "presentation" or a "specification" of the theory. Duval calls it a specification. In a given logic, every specification generates a theory, while every theory may be seen as a (completed) specification. In categorical terms, this means that the category $T$ of theories is a reflective subcategory of the category $S$ of specifications. This means that there is an adjunction between $S$ and $T$, such that the right adjoint is full and faithful. The right adjoint $R: T \rightarrow S$ allows us to consider a theory as a specification, the left adjoint $L: S \rightarrow T$ generates a theory from a specification, and the fact that $R$ is full and faithful means that each theory is saturated. In this case inference rules can be seen as cospans in $S$, and application as a pushout based construction. If the co-span representing an inference rule can be extended to a pushout square, then the application of an inference rule is the same categorical construction as the application of a graph rewrite rule. This line of work stems from the study of the semantics of computational effects in programming languages and it has been influenced by Ehresmann's theory of sketches [7], Makkai 'sketch entailments' [15-17], by the theory of institutions [9], as well as by the use of monads in computer science [20]. A fair criticism of this line of work that needs to be addressed in the future is the feasibility of computing with this approach.

Ochs' paper attempts to address central considerations of mathematical intuition and methodology. While it is true that to say that "it is impossible to talk about what mathematical intuition is" is to take the easy way out and, although most have taken this easy way, some (mostly philosophers) have laboured mightily to try to make sense of mathematical intuition and this paper does this for categorical constructions. One especially interesting 
feature of this paper is the discussion of archetypal models. After all, a motivation in the development of category theory was to give a precise statement of previously informal uses of the word 'universal' in this sense. The author makes some headway into the notion of what an archetypal model ought to be as a synthesis of notation and technique which is 'complete for reconstruction' in the sense of the paper. The author regards operations that discard information, like specializing to a particular case or dropping the intermediate steps of a proof, as 'projections', and operations that reconstruct information as 'liftings'. By working with several projections in parallel he tries to make sense of statements like "Set is the archetypal Cartesian Closed Category", as meaning that proofs about CCCs can be done in the "archetypal language" and then lifted to proofs in the general setting. He then proceeds to give examples of such projections, lifting and "archetypal" models. Thus there are at the moment, no theorems: instead, the paper shows several ways to represent constructions, and to structure proofs in several layers, capturing (one hopes) the paradigmatic models of reasoning in category theory. While this is a departure from traditional categorical logic, it seems to us that this kind of deep self-analysis needs space in the published literature, in the spirit of pluralism of methodologies within our field.

The paper by Baruss and Wood closes the volume with a small, but niceto-have result. They show that a first-order Kripke-Joyal model over a small category $\mathbf{C}$ can be converted into an elementarily equivalent model where the base category is a Heyting algebra (made up of maximal cribles of $\mathbf{C}$ ). At first sight the result looks very surprising: normally categories cannot be exchanged with complete Heyting algebras, a very special kind of the structure. But here this is to be somewhat expected, as we know that if we are dealing with intuitionistic logic using provability only (as opposed to modeling different proofs) then "complete Heyting algebras" are the only structures we can distinguish. The extra generality of a category will not get you any new information.

As the authors explain, the reason one can always use a complete Heyting algebra as a domain of forcing "lies in the construction of the derived category". In the derived category, each object of the original category indexes all future states of that object, so that any pathway from the past to the future in the original category can be traced along any of a number of pathways in the derived category. In that sense this is an "embedding theorem" whereby a general construction is multiply enfolded in a construction with more structure. Actually this is a paraphrasing explanation of how a Kripke model corresponds to a complete Heyting algebra for intuitionistic theories, which is what Joyal invented Kripke-Joyal semantics for. But this result has no bearing on different kinds of categorical semantics, where either proofs are modeled by morphisms or different structures are required (for example when modeling a substructural logic).

Tentative Conclusions. We fear that many of the heavily worked on problems on Categorical Logic did not make it into this special issue. Looking at this as a half full glass problem, we hope to organize more workshops and more special issues where these other lines of research are discussed. 
Most of the lines of investigation discussed above are still open, with new results and new application areas appearing all the time. Tempting as it is to speculate on the most promising areas for future investigation, we will resist temptation here and simply finish hoping for more meetings dedicated to the theme, as this seems clearly to have more to offer.

We should like to thank all the authors for their excellent contributions and the reviewers for their responsible and extended assistance in the selection and reviewing process. We are particularly grateful to Jean-Yves Beziau for his support in making this special issue possible in the first place.

\section{References}

[1] Bell, J.: The development of categorical logic. In: Gabbay, D. (ed.) The Handbook of Philosophical Logic, vol. 12, p. 279 (2005)

[2] Blass, A.R., Scedrov, A.: Freyd's models for the independence of the axiom of choice. Memoirs Am. Math. Soc. 79(404), 134 (1989)

[3] De Groote, Ph. (ed.): The curry-howard isomorphism. In: Cahiers du Centre de Logique, Université catholique de Louvain, Louvain-La-Neuve, vol. 8, p. 364 (1995)

[4] Corradini, A., et al.: Algebraic approaches to graph transformation. In: Rozenberg, G. (ed.) Handbook of Graph Grammars and Computing by Graph Transformation, pp. 163-312 (1997)

[5] Voevodsky, et al.: Homotopy Type Theory: Univalent Foundations of Mathematics. Princeton Institute for Advanced Study, Princeton (2013)

[6] Coecke, B. (ed.): New Structures for physics. In: Lecture Notes in Physics. Springer, Heidelberg (2011)

[7] Ehresmann, Ch.: Catégories et structures. Dunod, Paris (1965)

[8] Freyd, P.J.: The axiom of choice. J. Pure Appl. Algebra 19, 103-125 (1980)

[9] Goguen, J.A., Burstall, R.M.: Institutions: abstract model theory for specification and programming. J. ACM 39(1), 95-146 (1992)

[10] Jacobs, B.: Categorical Logic and Type Theory (Studies in Logic and the Foundations of Mathematics, vol. 141. Elsevier, North Holland (1999)

[11] Lawvere, F.W.: Functorial Semantics of Algebraic Theories. Ph.D. Columbia University, New York (1963)

[12] Lawvere, F.W.: An elementary theory of the category of sets. Proc. Nat. Acad. Sci. USA 52, 1506-1511 (1964)

[13] Lawvere, F.W.: Equality in hyperdoctrines and comprehension schema as an adjoint functor. Applications of Categorical Algebra (Proc. Sympos. Pure Math., vol. XVII, New York, 1968), pp. 1-14 (1970)

[14] Landry, E., Marquis, J.-P.: Categories in context: historical, foundational, and philosophical. Philos. Math. 13(1), 1-43 (2005)

[15] Makkai, M.: Generalized sketches as a framework for completeness theorems (i). J. Pure Appl. Algebra 115, 49-79 (1997)

[16] Makkai, M.: Generalized sketches as a framework for completeness theorems (ii). J. Pure Appl. Algebra 115, 179-212 (1997) 
[17] Makkai, M.: Generalized sketches as a framework for completeness theorems (iii). J. Pure Appl. Algebra 115, 241-274 (1997)

[18] Marquis, J.-P., Reyes, G.E.: The history of categorical logic: 1963-1977. In: Kanamori, A. (ed.) Handbook of the History of Logic: Sets and Extensions in the Twentieth Century, vol. 6, pp. 689-800 (2012)

[19] MacLane, S., Moerdijk, I.: Sheaves in Geometry and Logic: A First Introduction to Topos Theory. Springer, New York (1992)

[20] Moggi, E.: Notions of computation and monads. Inf. Comput. 93, 55-92 (1989)

[21] Rodin, A.: Axiomatic Method and Category Theory, Synthese Library, vol. 364. Springer, Berlin (2013)

[22] Sørensen, M.H., Urzyczyn, P.: Lectures on the Curry-Howard isomorphism, vol. 149. Elsevier, New York (2006)

[23] Vickers, S.: Topology via Logic. Cambridge University Press, Cambridge (1996)

Valeria de Paiva

Nuance Communications, California, USA

e-mail: valeria.depaiva@gmail.com

Andrei Rodin

Institute of Philosophy, Saint-Petersburg University

Russian Academy of Sciences, Moscow, Russia

e-mail: andrei@philomatica.org 